\title{
Clinical Characterization and Frequency of Observation of Hereditary Retinal Diseases: Multicentric Study in
} Panama in 2012-2013

\author{
Juan Manuel Muñoz Cuéllar and Rosa María Coco Martín \\ Institute of Oftalmo-Biología Aplicada (IOBA), University of Valladolid, Valladolid 47011, Spain
}

\begin{abstract}
Retinal dystrophies are genetically determined diseases, implying the loss of function of the retina with a wide phenotypic and genotypic variability. There are very few phenotypic, genotypic and epidemiological data on retinal dystrophies in Latin America. The Objective of this study is to describe the epidemiological and clinical characteristics of hereditary retinal and choroidal diseases, in retina practices in Panama. A descriptive study, from 2012 to 2013, was performed in the main retina practices in Panama. All detected patients were given a free appointment to gather their phenotypic characteristics and pedigrees. An incidence of five new cases per year, and an accumulated incidence of 5.35 patients per 10,000 was calculated for the public hospitals. A frequency of 2.7 cases per 1,000 patients was observed in the main retina practices, where $69 \%$ had rod-cone dystrophies, $14.3 \%$ cone-rod dystrophies, $7.1 \%$ Stargardt disease, $4.8 \%$ Stargardt-like macular dystrophy and two patients presented other dystrophies. Blindness was the main family antecedent (45.2\%). Retinal pigment was present in $59 \%$ and strabismus in $21.4 \%$ of the patients. Rod-cone and cone-rod dystrophies had similar geographic distribution and the autosomal recessive inheritance pattern was the most frequently observed. This study gives the first phenotypic data of retinal dystrophies in Panama to orient clinicians for a better diagnosis and phenotyping-genotyping correlation for retinal dystrophies in Central America.
\end{abstract}

Key words: Retinal dystrophies, retinitis pigmentosa, rod-cone dystrophy, cone-rod dystrophy, Stargardt disease, phenotypic characterization.

\section{Introduction}

Retinal dystrophy is a heterogeneous group of hereditary diseases of genetic origin, affecting the retinal pigment epithelium-photoreceptor complex [1] and the transduction process from light to electricity. The large amount of biochemical reactions involved in the transduction process makes them vulnerable to mutations in the genes coding for the synthesis of enzymes that participates in the process. This biochemical complexity can produce similar phenotypic presentations from different mutations and diversity in the clinical presentations of a mutation itself [2].

Corresponding author: Juan Manuel Muñoz Cuéllar, doctoral candidate, retina specialist, research fields: visual sciences and retinal diseases.

\subsection{Epidemiology}

Even though hereditary diseases of the retina are individually rare, the sum of their prevalence impacts as a major cause of disability. They are often progressive, leading to visual loss and even blindness. Hereditary dystrophies of the retina have an estimated prevalence of $1: 1,490$ in France [4], what could be extrapolated to the Panamanian population $(4,000,000$ inhabitants) representing approximately 2,600 affected. RP (retinitis pigmentosa) has an estimated prevalence between 1:3,000 and 1:5,000, and affects at least two million people in the world, apart from other hereditary diseases of the retina [5].

Retinal dystrophies are characterized by degeneration of the photoreceptors [1] that can also be secondary to degeneration of the choriocapillaris [6] 
or retinal pigment epithelium [7], as parts of an interrelated functional unit. The retina can also be degenerated by alterations in its cellular stabilization and organization [8].

\subsection{Genetic and Phenotypic Heterogeneity}

Retinal dystrophies are transmitted in simple Mendelian inheritance, by alterations in a particular gene [9], although there are forms of digenic inheritance.

Genotypic heterogeneity refers to the similarity among phenotypes with total or partial overlapping. At the moment, there are at least 18 genes linked to Leber congenital amaurosis [10], six genes linked to progressive cones dystrophy [11] and 23 genes linked to rod-cone dystrophy [12]. In Stargardt Disease 1, all mutations practically occur in the gene of the ABCA4 (ATP (adenosine triphosphate) binding cassette 4 subfamily). Around $70 \%$ of patients with X-linked RP have mutations in the RPGR (retinitis pigmentosa GTPase (guanosine triphosphatase) regulator gene), and a small percentage have mutations in the RP2 (retinitis pigmentosa 2 gene). The most extreme example of genetic heterogeneity is the autosomal recessive RP, with 25 genes described and many still not identified.

On the other hand, the phenotypic heterogeneity refers to similar mutations with unexplained differences in the phenotypes, probably associated with environmental factors, other genes modifying the disease or epigenetics phenomena [13]. An example is the mutation in the ABCA4 gene producing Stargardt Disease 1, fundus flavimaculatus, cone-rod or rod-cone dystrophies. Other examples are the mutations in the genes encoding the CRB1 (Crumbs Homolog 1), LRAT (lecithin retinol acyl transferase), the tyrosine-kinase MER receptor, RPE65 (Retinal pigment epithelium 65), the SPATA7 (spermatogenesis-associated protein 7) and the TULP1 (Tubby-like protein). Mutations in these genes can produce Leber congenital amaurosis or early RP.
It is worth mentioning that even different diseases can have clinical overlapping. For example, patients with STGD1 (Stargardt Disease 1) show defects in the mid-peripheral visual field with the advance of age, similar to patients with cone-rod dystrophies, while patients with cone dystrophy are very difficult to distinguish from patients with cone-rod dystrophies [14].

\subsection{Clinical Diagnosis}

The initial approach of patients with hereditary retinal diseases is the categorization into rod-cone or cone-rod dystrophy, since the two involve different evolutions and prognosis. Rod-cone dystrophies tend to affect the entire vision along the years. Cone-rod dystrophies initially affect the central vision. It is important to determine the sub-type for conducting a proper advice and to offer potential treatments. The clinical history should ask for night vision problems [15, 16] and tubular vision [9]. The clinical diagnosis can be supported by complementary tests like visual fields, dark adaptation test, color vision, glare tests [17], electroretinogram, fluorangiography, autofluorescence or optical coherence tomography [18].

\subsection{Genetic Diagnosis}

Genetic diagnosis is performed in cases of autosomal dominant, recessive, or X-linked, to improve the genetic advice and avoid the appearance of new cases in the family. Simplex forms refer to cases in which no other family members have been identified with the diseases and can correspond to cases of autosomal recessive, X-linked, autosomal dominant, or "de novo" mutations [19]. The genetic diagnosis is also used to confirm a previous diagnosis and is gaining importance with the advent of gene therapy and the possibility of specific treatments. It is necessary to identify new genes and describe the genotypic-phenotypic correlations in order to develop genetics based treatments [20]. Moreover, more 
detailed knowledge is needed to understand the interaction among different proteins involved.

The screening for mutations and phenotypes can provide complex data to be analyzed. Each mutant gene can contain different mutations requiring extensive analysis to determine which mutation causes the phenotype in study. This problem can be tackled with computational methods of analysis; which can provide "phenotypic sequence" and reduce the costs of laboratory tests [21]. The massive sequencing is assuming an important role in the genetic diagnostic approach [22]. The best time to inform about the different aspects of the disease is before childbearing, for what could be considered the use of a DNA (deoxyribonucleic acid) bank, since the technology for detection may continue to improve in the future [23].

Even though genetic diseases are a major cause of blindness in children, there is very little information about them in developing and newly industrialized countries [3].

A Brazilian study in children found that retinal diseases were the most frequent cause of visual disability [24], however, some of them are not inherited [25].

There are no Panamanian publications about retinal dystrophies, and very few Latin-American publications can be found in PubMed.

\section{Materials and Methods}

A prospective observational study has been conducted in patients attending the retina practices, from January 2012 to December 2013.

All patients with diagnosis of retinal dystrophy, in the two public hospitals having retina practices in Panama, were included for the calculation of incidence rates throughout the period of the study.

Patients detected with retinal dystrophies at the two public hospitals and at the main private retina practice were invited to have a more detailed evaluation in a free appointment to perform a clinical and epidemiological descriptive study.
Clinical histories were directed to clinical presentation, symptoms and evolution of the retinal dystrophy. It included the construction of a pedigree and a subsequent classification of the inheritance pattern.

An ocular examination was performed, including VA (visual acuity), refraction, IOP (intraocular pressure), bio-microscopy, eye fundus, contrast sensitivity test, colors vision test and visual fields. Optical coherence tomography, fluorangiography and electroretinography were also taken, if needed. The statistical analysis was performed using a Microsoft Excel database.

The study included patients with previous or "de novo" diagnosis of retinal dystrophy, capable of understanding and accepting their participation in the study, and that were able to attend the detailed clinical examination. Patients without any signs or symptoms of retinal dystrophy were excluded.

The study was carried out adhered to the principles of the Helsinki Declaration, and all patients passed through an informed consent process.

\section{Results}

\subsection{Study of Incidence}

In the study period, from 2012 to 2013, a total of 18,684 patients were attended in the two public hospitals for adults, with retina practice in Panama.

Ten patients were diagnosed "de novo", for an incidence of five newly diagnosed patients per year, and an accumulated incidence of 5.35 patients with hereditary retinal dystrophies for every 10,000 patients in the retina practices. Seven out of the 10 newly diagnosed patients were evaluated because of retinal dystrophy in a family member. Two of them presented nictalopia as their primary symptom, and only one patient presented visual loss at the time of diagnosis.

The incidence of typical RP was 1:10,000 and the overall incidence of rod-cone dystrophies, including atypical forms of RP was 2.67:10,000 patients. 


\subsection{Descriptive Study}

The main private retina practice attended 3,420 patients along the two years, which added to the 18,684 attended in the public services, totaled 22,104 patients.

Sixty patients with retinal dystrophies from 49 families were identified in the three clinics, corresponding to a frequency of 2.7 cases for every 1,000 patients.

Forty two patients (23 males and 19 females) were attended in a detailed clinical evaluation, and their mean age was $37 \pm 19.64$ years old.

Organized retinal pigment was present in 25 , and strabismus in nine patients.

Twenty five patients had rod-cone dystrophies, distributed as follows: 20 patients with RP, two with retinitis punctata albescens, and five with rod-cone dystrophy without pigment. Rod-cone dystrophy was also found in two patients with Usher syndrome, as the only syndromic form found. Six patients presented cone-rod dystrophies, two patients had Stargardt disease, fundus flavimaculatus was found in one patient, two patients presented Stargardt-like macular dystrophy, one patient had central choroidal areolar dystrophy, and another patient had congenital stationary night blindness.

Twenty two patients $(52.3 \%)$ presented autosomal recessive inheritance pattern, and 11 cases $(25.5 \%)$ did not have an identifiable inheritance pattern or enough information of their family history.

Relative consanguinity was present in 24 patients (57\%), understanding as relative consanguinity the status of descendants of father and mother from the same village in a rural area. Blindness was present in 19 of the 42 patients, as family antecedent. The geographical distribution was similar for rod-cone and cone-rod dystrophies.

A mean VA of $0.6 \pm 0.7$ LogMAR (logarithm of the minimum angle of resolution) was found for the 10 patients $(23.8 \%)$ with new diagnosis.

\subsubsection{Rod-Cone dystrophies}

Twenty nine patients (17 males and 12 females) presented rod-cone dystrophies, with a mean age of $38.46 \pm 20.28$ years old.

Two of the patients presented bilateral hearing loss, and fit in the diagnosis of Usher II representing 4.76\% of the studied population.

\subsubsection{Clinical History}

The family history was positive in 18 patients, with blindness in 14 (48.3\%) patients, juvenile cataracts in two, retinal dystrophy in seven, retinal detachment in one, pathological myopia in one, and glaucoma in two patients. Relative consanguinity was present in 15 patients from 14 families. The inheritance pattern was autosomal dominant in six patients (20.7\%) from five families, autosomal recessive in $10(34.5 \%)$ from different families, and X-linked in five (17.5\%) from two families. The inheritance pattern could not be identified in eight patients (27.6\%) from six families. One patient presented ophthalmic antecedent of Pseudo-Coats, with amaurosis in one eye, and photo-coagulation scars in the other eye. Four patients had history of strabismus, two of retinal detachment, one of cystic macular edema, one of glaucoma, and one of amblyopia. Nineteen patients referred visual loss as the main reason for consultation. Two consulted for central fotopsias, four for nictalopia, and four for family history of retinal dystrophies.

The mean age of diagnosis was $26.3 \pm 18.9$ years old, and the symptoms began at a mean age of $24.44 \pm$ 16.9 years old.

The first symptom to appear was: nictalopia, in 17 patients, hemeralopia in nine, visual field contraction in two, and photophobia in one patient. All of the cases were progressive.

\subsubsection{Ophthalmic Examination}

Seventeen patients were myopic, four were hyperopes, and eight were close to emetropia. The mean spherical equivalent was of $-1.86 \pm 3.25$ diopters. The mean VA was $0.86 \pm 0.7$ LogMAR, where three eyes with vision equal or below light 
perception were not included in the statistical analysis.

The mean IOP was $12.5 \pm 3.3 \mathrm{mmHg}$. The ocular motility was altered in a patient with exophoria another with esophoria and two with exotropia. Four patients presented lenses with cortico-nuclear cataracts, and two with posterior subcapsular cataracts. Fifteen of the 58 eyes were pseudoaphakic, and the rest of the eyes had clear lenses. Optic nerve atrophy was found in three eyes of two patients, and whitish gliosis in the two eyes of another patient.

The retinal vessels were thinned in 20 (68.9\%) of the 29 patients. Twenty one of the 29 patients $(72.4 \%)$ had organized pigmentation in the mid-peripheral retina, rounded in seven cases, and spiculated in 14 .

Two patients (6.9\% of the rod-cone dystrophies) presented retinitis punctata albescens. Three of the non-pigmented cases had salt and pepper eye fundus appearance. One patient had choroidal atrophy, and two had normal appearance of the eye fundus.

\subsubsection{Other Visual Examinations}

Ten patients had normal color vision, and 19 presented dyschromatopsia. The total color blindness was the most common dyschromatopsia (11 cases), and deuteranomaly was found in seven cases. One patient could not be assessed due to profound visual loss. The mean contrast vision in Pelli-Robson score was 1.756, among the 24 eyes with a VA of 0.47 LogMAR or better. The visual field was found contracted in 28 , and could not be evaluated in one patient.

\subsubsection{Cone-Rod Dystrophies}

Six patients with cone-rod dystrophy (three males and three females) were found, all of them from different families, and already diagnosed with retinal dystrophy.

The mean age was $28 \pm 16.54$ years old. No difference was noted between the age of patients with rod-cone and cone-rod dystrophies (probability of occurrence $p=0.71$ ).

\subsubsection{Clinical History}

Four patients $(67 \%)$ referred nictalopia, and the other two (33\%) referred hemeralopia as the first symptom. The mean age of onset was $17 \pm 15.19$ years. Patients with nictalopia as first symptom also had central photopsias, and were younger than those who began with hemeralopia (22.7 vs. 33 years old). All of the patients consulted for progressive visual loss.

The family history was positive for blindness in three patients (50\%), rod-cone dystrophy (a sibling) in one, glaucoma in one, and strabismus in another patient.

The inheritance pattern was autosomal recessive in four patients, autosomal dominant in one, and one case remained undetermined.

\subsubsection{Ophthalmic Examination}

One patient presented myopia ( -3.00 diopters), and the other five were close to emetropia.

The mean VA was $1.4 \pm 0.6$ LogMAR. A patient without light perception was not included in the statistical analysis.

The mean IOP was $11.2 \pm 3.6 \mathrm{mmHg}$. The ocular motility was altered in three patients. Exophoria was present in one, and exotropia in two patients. All the three patients came from different families and different provinces.

Two eyes of one patient had posterior subcapsular cataract, two eyes of another were pseudophakic. The rest of the lenses were clear.

All of the optic nerves had normal appearance.

The retinal vessels were thinned in four patients, two of them with pigmented spicules.

Three of the patients presented organized pigment in the eye fundus, concentrated in the posterior pole. The other three had salt and pepper fundus appearance.

\subsubsection{Other Visual Examinations}

The color vision was altered in three patients with complete color blindness in two and deuteranomaly in the other. The profound visual loss of the other three patients did not permit their evaluation. It was possible to assess the contrast sensitivity in three 
patients, showing a median of $0.8 \pm 0.38$. The visual fields presented central scotoma with contraction of the peripheral field in five cases. The sixth case was amaurotic in both eyes.

3.2.3. Stargardt Disease and Fundus Flavimaculatus

The different forms of Stargardt and Stargardt-like were present in five patients with a mean age of $46.6 \pm$ 21.42 years old.

The age of onset of symptoms was similar between Stargardt and Stargardt-like dystrophy $(p=0.46)$, and also similar between Stargardt disease and fundus flavimaculatus $(p=0.20)$.

\subsubsection{Stargardt Disease}

Stargardt disease was present in two patients (12 and 56 years old).

One of the patients had antecedent of abnormal electroencephalographic waves and arachnoid cysts. The mean age of perceived visual decline was 30 years old.

The mean VA was 1 LogMAR (range between 1.3 and 0.7 LogMAR).

The IOP were within normal limits. Cortical cataracts were present in one patients, and exotropia in one patient.

The optic nerve head was normal in both patients, with arteriolar thinning in one of them.

Mid peripheral flecks with metallic aspect of the macula were observed in one patient, and diffuse pigmentary changes were observed in the other. The color vision was altered (deuteranomaly) in one of the patients, and the Pelli-Robson scores were between 1.2 and 1.5. Mild visual field contraction was found in both cases (both of them with at least $40^{\circ}$ ).

The two cases corresponded to autosomal recessive inheritance patterns.

\subsubsection{Fundus Flavimaculatus}

Fundus flavimaculatus was found in a 61-year-old female who came for a routine ophthalmic examination. The patient referred family history of blindness.

Her mean VA was 0.15 LogMAR, and the IOP were within normal limits. The lenses presented cortico-nuclear cataracts, with normal ocular motility and normal optic nerve appearance. The eye fundus presented arteriolar thinning with yellowish pigmentary changes in the perifoveal area. The contrast sensitivity and color vision were normal, although the perimetry showed an arcuate defect. The inheritance pattern could not be determined.

\subsubsection{Stargardt-Like Macular Dystrophy}

Stargardt-like macular dystrophy was identified in two cases (63 and 40 years old) which were father and daughter from the same family. One of them had decreased vision and previous diagnosis. The mean refraction was +2.25 diopters. The 63 years old patient presented hearing loss, and both had family history of Strabismus and glaucoma. The mean VA was 0.37 LogMAR. The eye pressure was found within normal limits. Nuclear cataracts, optic nerve gliosis and rounded flecks in the retina were present in the oldest patient, and diffuse pigmentary changes were observed in the other. The ocular motility was normal in both cases. One of the patients was color blind, and the other had red-green abnormality. The contrast sensitivity was altered in both cases (Pelli-Robson score between 0.75 and 1.65). Both cases had central scotoma, with mild visual field contraction in one of the cases. An autosomal dominant inheritance pattern was identified.

\subsubsection{Other Dystrophies}

\subsubsection{Central Areolar Choroidal Dystrophy}

Central areolar choroidal dystrophy was found in a 56-year-old male, who referred glare as the first symptom, associated with visual field contraction and central photopsias. The VA was 1.78 LogMAR in both eyes, with IOP of $10 \mathrm{mmHg}$. The lens was clear in both eyes, the optic nerves were excavated, and the retinal arterioles were thinned. Drusen were not observed in the eye fundus, and the choroidal atrophy reached the optic nerve. The color vision was altered (deuteranomaly), as well as the contrast sensitivity and the visual field (contraction and central scotoma). The 
patient denied any family history of ocular diseases, and it was not possible to establish an inheritance pattern.

\subsubsection{Congenital Stationary Night Blindness}

Congenital stationary night blindness was found in a 20-year-old male, with pathological history of seizures during childhood. The patient had antecedent of exophoria, nystagmus, and refraction near emetropia in both eyes. The first visual symptom was hemeralopia since age 6 . The VA was 0.4 and 0.5 LogMAR. Whitish dots were found in the extreme periphery of the lower retina of both eyes. The color vision was preserved, as well as the contrast sensitivity. The visual fields were contracted in both eyes. The inheritance pattern could not be identified. The scotopic electroretinogram showed a normal " $a$ " wave, and a "b" wave with decreased amplitude.

\section{Discussion}

New cases were mainly identified by their family history $(70 \%)$, and contrary to expected, visual loss was the less common complaint driving to a new diagnosis. Blindness was the main family antecedent found.

The VA was scored in LogMAR for statistical purposes [26]. The contrast sensitivity was measured with the Pelli-Robson test in order to evaluate patients with different VA [27]. Red-green color deficiencies were mainly expected [28], and the Ishihara test is more accurate than the Farnsworth test to evaluate them [29]. The Goldmann perimetry was preferred over the automated for its indication in low vision patients [30].

The retinal dystrophies frequency in 2.7 per 1,000 patients in the retina practices was higher than found in the Danish population $(1: 3,454)$, higher than observed in children in Denmark $(13: 100,000)$ [31], and lower than the frequency of RP observed in Puerto Rico (44 in 10,100 people examined) [32]. A high frequency was expected, since the studied population already had retinal diseases.
The frequency of RP (30.95\%) was proportionally lower than reported $(47 \%)$, and the frequency of syndromic forms $(4.8 \%)$ was similar to that observed in the south of France [33]. The proportion of cases with autosomal recessive inheritance (53\%) was greater, while the proportion of simplex cases $(25.5 \%)$ was lower than described in the Danish population (23\% and $45 \%$, respectively) [34].

It was expected to find a higher proportion of male patients, because of the X-linked cases. The high frequency of relative consanguinity is an expected result, since the autosomal recessive is the most common inheritance pattern [35].

The most common retinal dystrophy was the rod-cone dystrophy, as expected. The higher frequency of cone-rod dystrophy when compared to Stargardt disease contradicts the literature [35].

The frequency of pigmented cases was lower than described in the south of France (59\% vs. $70 \%$ ) [33].

\subsection{Rod-Cone Dystrophies}

The greater frequency of males (59\%) is not far from the 56.8\% reported in Puerto Rico [32], and the presence of two cases with Usher Syndrome II (4.76\% of the total) is consistent with the literature [35].

The wide range in the age of beginning of symptoms and diagnosis was expected, given the phenotypic variability, as patients with autosomal dominant forms use to have a latter onset, compared to $\mathrm{X}$-linked forms, which use to initiate symptoms in the first decade of life [36].

The mean age of onset of symptoms was higher than found in consanguineous marriages [37]. This could be attributed to the presence of autosomal dominant added to a majority of autosomal recessive cases, as expected [35]. The proportion of X-linked cases (17.2\%) was slightly higher than described in Puerto Rico (9\%) [32].

Strabismus as the main ophthalmic antecedent, present in $13 \%$ of the patients with rod-cone dystrophies seemed to be higher than in the general population (4\%) [38]; However, it is not very different 
from what has been reported in RP (18\%) in Puerto Rico [32], probably due to macular malfunction [39] and disappearance of the fusion stimulus.

The higher frequency of myopia compared to hyperopia coincides with the literature [35], and has been related with alterations in the metabolism of dopamine [40].

Visual loss as the main cause of consultation was expected, as it compromises the quality of life.

Symptoms related to poor night vision and visual field contraction coincided with the literature. The presence of hemeralopia or photophobia in $34.5 \%$ of the patients can be attributed to a greater dispersion of intraocular light, even in the absence of observable cataract [41], or to cystic macular edema, described in $18 \%$ of RP patients [42].

The mean VA of 0.14 (metric decimal) is within the range described by the literature ( 0.3 to 0.05 metric decimal) [32].

It was expected to find normal IOP as it has not been described that rod-cones dystrophies increase it. The frequency of cataracts and pseudophakia (32.8\%) was expected, since patients with RP tend to develop early cataracts [35].

The proportion of optic nerves with normal appearance (89.6\%) was unexpected, and higher than described (62.8\%), although the pigmentation of the patients may have influenced the color of the optic nerve head [43].

The vascular thinning present in $69 \%$ of the patients coincides with the literature, as it is expected in typical forms, even preceding the appearance of retinal pigmentation [35], and was close to the frequency observed in Puerto Rico (65.9\%) [32].

A high frequency of organized pigment (72\%) was expected and did not differ too much from the literature (81.4\%) [32].

The color vision altered in only $34.4 \%$ of the patients can be attributed to a mild affectation of cones in some of the cases; and not to their ages, since no difference was found between cases with normal color vision and the rest of the rod-cone dystrophies. The $27 \%$ of cases with complete color blindness; might be due to more advanced diseases and more severe phenotypic presentations.

The contrast sensitivity score was above normal for 60 years old patients (1.65), below expected for young patients [44, 45], and suggests that it should always be compared within the same age group.

The severe visual field contraction suggested advanced stages of the disease [35] and was a common denominator for all patients.

\subsection{Cone-Rod Dystrophies}

It was expected to have an equal involvement of men and women, since there were no X-linked cases. The autosomal recessive pattern was observed more often than expected, and contradicts the previous literature indicating that the autosomal dominant pattern is the most common form [35].

The similarity in geographical distribution when compared with rod-cone dystrophies raises the possibility of both dystrophies been caused by mutations in the ABCA4 gene, as the main identified cause of autosomal recessive cone-rod dystrophy [16] associated with Stargardt disease and rod-cone dystrophies [46], and reaching a frequency of $10 \%$ in the general population [47]. The decrease in the VA as the main reason for consultation is an expected result as it produces disability.

There is not an expected prevalence of strabismus, even though there are cases of strabismus associated with mutations of the KCNV2 (potassium voltage-gated channel modifier subfamily $\mathrm{V}$ member 2) gene. The proportion of strabismus in patients with cone-rod dystrophy $(50 \%)$ is significantly higher ( $p=0.026)$ that in rod-cone dystrophies $(13 \%)$, and might have a sensory origin [39].

The mechanism generating myopia in cone-rod dystrophies could be similar to that occurring in rod-cone dystrophies [40].

It was unexpected to find $67 \%$ of the patients 
debuting with nictalopia instead of hemeralopia (present in only 33\% as first symptom) [35]. It is worth mentioning that all patients presenting nictalopia as initial symptom referred central photopsias as well, and that photopsias were not present in any of the patients debuting with hemeralopia. Central photopsias seemed to appear in more advanced stages of more severe diseases, and patients presenting it were 10 years younger. Nictalopia probably appeared as first symptom when the cones were already altered since very early in life.

Hypothyroidism in one patient can be explained by its greater frequency reported in patients with retinal dystrophies and their families [48].

Blindness as the main family antecedent could be related with the severity of the mutation.

The low VA was expected [49], as it was a normal mean eye pressure.

The normal optic nerve in all cases, and high frequency of vascular thinning (66.7\%), were unexpected results that contradict the literature. The deposits of pigment in the posterior pole are well described in the literature [49].

It was expected to find color vision abnormalities, as the disease involved the cones primarily. The altered contrast sensitivity, and visual field contraction with central scotoma were expected results [16].

\subsection{Stargardt Disease, Fundus Flavimaculatus and Stargardt-Like Macular Dystrophy}

Even though Stargardt disease was expected to be more common than cone-rod dystrophies [35], the proportion of Stargardt disease (7\%) is not far from that reported in the literature $[33,47]$.

The two cases of Stargardt-like macular dystrophy were more probably associated with mutations in the ELOVL4 (elongation of very long chain fatty acids 4) [50] or PROM1 (Prominin 1) genes [47].

The proportion of Stargardt-like macular dystrophies probably distorted because of two individuals from the same family, and the small size of the studied population [47].

The presence of cataract above age 55 was higher than estimated by the National Eye Institute in the normal American population (100\% vs. 16.19\%, respectively) [51].

\subsubsection{Stargardt Disease}

The case of maculopathy in one family could be an affected individual, or a case of age-related macular degeneration in a heterozygous.

The abnormal electroencephalography in a patient can be related to the role in lipids transport played by the ABCA4 gene in the brain [52].

The presence of nictalopia is not common but is well described as a feature of classic Stargardt disease [53]. The VA between 0.05 and 0.2 (metric decimal) and red-green abnormality in the color vision of one of the patients were expected results [35, 47]. The mean age of perceived visual decline, was higher than that found in Tunisia (8.1 years) [54].

The refractive defects were within the range described by Mäntyjärv and Tuppurainen [55] (-8.50 to +2.25 ), and the mean spherical equivalent of -0.75 diopters is consistent with the literature [56]. Exotropia was noted in the patient with the lowest VA (0.08 and 0.05 metric decimal), which suggests a sensory origin of the strabismus [39].

The color vision was expected to be abnormal, and the normal color vision in the oldest patient was probably due to a late onset disease.

The diminished contrast sensitivity was an expected finding [57], as it was the mild visual field contraction [53] and the diminished electroretinographic amplitude [58].

\subsubsection{Stargardt-Like Macular Dystrophy}

The father to daughter transmission coincides with previous observations of no father to son transmission [59].

The presence of hemeralopia and photophobia was expected due to cone malfunction.

Stargardt-like patients also had refractions within the range described in the literature [55], although the 
mean spherical equivalent was hyperopic $(+1.12)$ [56].

Cases of strabismus in the family history can not be related with the affected gene.

The more affected color vision was expected in the older patient, as the disease progresses from alteration in the red-green to the blue-yellow axis [60].

The central scotoma with mild visual field contraction was an expected result [61].

\subsection{Other Retinal Dystrophies}

\subsubsection{Central Areolar Choroidal Dystrophy}

Glare was expected, associated with affectation of the macula, or subclinical subcapsular changes in the lens, even without evidence of cataract [41, 62].

The beginning of symptoms in the sixth decade was not far from what has been reported in the literature [35].

VA of 1.78 LogMAR was far below expected [35] and probably caused by the location of the foveal atrophy.

The red-green affectation of the color vision coincides with the literature [63], preceding alterations in contrast sensitivity [64].

The optic nerve excavation with visual field contraction and the vascular thinning were challenging, raising the possibility of coexisting glaucoma and hypertensive retinopathy. The disease was graded in Stage IV of Deutman.

It was unexpected to find a possible simplex case, since the disease is usually inherited in an autosomal dominant pattern linked to mutations in the chromosome 17p13 (short arm of chromosome 17) [65] and the peripherin/RD gene [66], even though autosomal recessive cases have been reported, and there are still genes to be identified [67].

\subsubsection{Congenital Stationary Night Blindness}

There are cases reported without nictalopia in patients that have never had a normal vision [68], or in Type 2 disease (rod's signal attenuated, but still present) [69]. Hemeralopia as the first symptom also coincides with the diagnosis of Type 2 disease [70].
It was expected to find myopia [35], fairly good vision, strabismus and nystagmus [71].

The white dots in the peripheral retina were unexpected, but their regional localization [72] and the presence of scotopic "b" wave in the electroretinogram contradict any diagnosis of fundus albipunctatus [73].

The normal color vision and mild visual field contraction were expected, although the normal contrast sensitivity was not $[71,74]$.

\section{Conclusions}

A high incidence of retinal dystrophies was found, with higher than expected age of onset of symptoms, and high frequency of strabismus. The geographical distributions resulted similar among them. Non-pigmented rod-cone dystrophies and autosomal recessive cone-rod dystrophies were unexpectedly frequent. The family history of blindness was the main fact driving to new diagnoses.

This study gives the first basis for a phenotypes database and further DNA bank, as well as clinical data to permit a better understanding of these diseases by visual health practitioners.

\section{Conflict of Interests}

The study was funded by the authors without any conflict of interests.

\section{References}

[1] Nentwich, M. M., and Rudolph, G. 2013. "Hereditary Retinal Eye Diseases in Childhood and Youth Affecting the Central Retina." Oman J. Ophthalmol. 6 (Suppl 1): S18-25.

[2] Castagnet, P., Mavlyutov, T., Cai, Y., Zhong, F., and Ferreira, P. 2003. "RPGRIP1s with Distinct Neuronal Localization and Biochemical Properties Associate Selectively with RanBP2 in Amacrine Neurons." Hum. Mol. Genet. 12 (15): 1847-63.

[3] Puech, B., Kostrubiec, B., Hache, J. C., and François, P. 1991. "Epidemiology and Prevalence of Hereditary Retinal Dystrophies in the Northern France." $J$. Fr. Ophtalmol. 14 (3): 153-64.

[4] Chizzolini, M., Galan, A., Milan, E., Sebastiani, A., 
Costagliola, C., and Parmeggiani F. 2011. "Good Epidemiologic Practice in Retinitis Pigmentosa: From Phenotyping to Biobanking." Curr. Genomics 12 (4): $260-6$

[5] OMIM (Online Mendelian Inheritance in Man). 1986. "Macular Dystrophy, Retinal, 1, North Carolina Type; MCDR1." Accessed October 22, 2014. http://www.omim.org/entry/136550.

[6] OMIM (Online Mendelian Inheritance in Man). 1986. "Macular Dystrophy, Patterned, 1; MDPT1." Accessed September 13, 2015. http://www.omim.org/entry/169150.

[7] Molday, R. S., Kellner, U., and Weber, B. H. F. 2012. "X-Linked Juvenile Retinoschisis: Clinical Diagnosis, Genetic Analysis, and Molecular Mechanisms." Prog. Retin. Eye Res. 31 (3): 195-212.

[8] Yanoff, M., and Duker, J. S. 2013. Ophthalmology: Expert Consult: Online and Print. Atlanta: Elsevier, Health Sciences.

[9] Orphanet. 2015. "Leber Congenital Amaurosis." Accessed January 20, 2015. http://www.orpha.net/consor/ cgi-bin/Disease_Search.php?lng=EN\&data_id=3243\&Di sease_Disease_Search_diseaseGroup=leber-congenital-am aurosis\&Disease_Disease_Search_diseaseType=Pat\&Dise ase(s)/group $\% 20 \mathrm{of} \% 20$ diseases=Leber-congenital-amauro sis\&title=Leber-congenital-amaurosis\&search=Disease_S earch_Simple.

[10] Orphanet. 2015. "Progressive Cone Dystrophy." Accessed January 20, 2015. http://www.orpha.net/consor/ cgi-bin/Disease_Search.php?lng=EN\&data_id=1842\&Dis ease_Disease_Search_diseaseGroup=cone-dystrophy\&Di sease_Disease_Search_diseaseType=Pat\&Disease(s)/gro up\%20of\%20diseases=-Progressive-cone-dystrophy\&title =Progressive-cone-dystrophy\&search=Disease_Search_S imple.

[11] Orphanet. 2007. "Cone Rod Dystrophy." Accessed October 26, 2014. http://www.orpha.net/consor/cgi-bin/ Disease_Search.php?lng=EN\&data_id=1843\&MISSING $\%$ 20CONTENT=Cone-rod-dystrophy\&search=Disease_S earch_Simple\&title=Cone-rod-dystrophy.

[12] Sociedad Española de Oftalmología. 2010. "VI.3. Distrofias Maculares". Accessed October 5, 2014. http://www.oftalmoseoformacion.com/wp-oftalmoseo/do cumentacion/p2010/Cap06-03.pdf.

[13] Den Hollander, A. I., Black, A., Bennett, J., and Cremers, F. P. M. 2011. "Lighting a Candle in the Dark: Advances in Genetics and Gene Therapy of Recessive Retinal Dystrophies." J. Clin. Invest. 120 (9): 3042-53.

[14] Ohba, N., and Ohba, A. 2006. "Nyctalopia and Hemeralopia: The Current Usage Trend in the Literature.” Br. J. Ophthalmol. 90 (12): 1548-9.

[15] Godara, P., Cooper, R. F., Sergouniotis, P. I., Diederichs, M. A., Streb, M. R., Genead, M. A., et al. 2012.
"Assessing Retinal Structure in Complete Congenital Stationary Night Blindness and Oguchi Disease." Am. J. Ophthalmol. 154 (6): 987-1001.

[16] Science Dictionary. 2012. "What is GLARE? Definition of GLARE (Science Dictionary). Science Dictionary. Accessed $\quad$ February 2, 2015. $\mathrm{http}: / /$ thesciencedictionary.org/glare/.

[17] ISCEV (International Society for Clinical Electrophysiology of Vision). 2001. "A Guide to Procedures.” Accessed January 21, 2014. http://www.iscev.org/standards/proceduresguide.html

[18] Schwartz, S. B., Aleman, T. S., Cideciyan, A. V., Swaroop, A., Jacobson, S. G., and Stone, E. M. 2003. "De Novo Mutation in the RP1 Gene (Arg677ter) Associated with Retinitis Pigmentosa." Investig. Opthalmology Vis. Sci. 44 (8): 3593-7.

[19] Foundation, N. 2004. Retinal Dystrophies: Functional Genomics to Gene Therapy. Hoboken: John Wiley \& Sons.

[20] Harper, M. A., Chen, Z., Toy, T., Machado, I. M. P., Nelson, S. F., Liao, J. C., et al. 2011. "Phenotype Sequencing: Identifying the Genes That Cause a Phenotype Directly from Pooled Sequencing of Independent Mutants." PLOS ONE 6 (2): e16517.

[21] Berg, J. S., Khoury, M. J., and Evans, J. P. 2011. "Deploying Whole Genome Sequencing in Clinical Practice and Public Health: Meeting the Challenge One Bin at a Time." Genet. Med. 13 (6) :499-504.

[22] Fahim, A. T., Daiger, S. P., and Weleber, R. G. 1993. "Retinitis Pigmentosa Overview." In: GeneReviews ${ }^{\circledR}$, edited by Pagon, R. A., Adam, M. P., Ardinger, H. H., Bird, T. D., Dolan, C. R, Fong, C. T., et al. Seattle (WA): University of Washington. Accessed September 7, 2014. http://www.ncbi.nlm.nih.gov/books/NBK1417/.

[23] American Academy of Ophthalmology. 2009. "Retinal Dystrophies of Childhood." Accessed January 21, 2014. http://www.aao.org/eyenet/article/childhood-retinal-dystr ophies.

[24] Haddad, M. A. O., Sei, M., Sampaio, M. W., Kara-José, N. 2007. "Causes of Visual impAirment in Children: A Study of 3,210 Cases." J. Pediatr. Ophthalmol. Strabismus. 44 (4): 232-40.

[25] Neto, E. C., Anele, E., Rubim, R., Brites, A., Schulte, J., Becker, D., et al. 2000. "High Prevalence of Congenital Toxoplasmosis in Brazil Estimated in a 3-year Prospective Neonatal Screening Study." Int. J. Epidemiol. 29 (5): 941-7.

[26] Touzeau, O. 2003. "Calculating the Mean Visual Acuity and the Change in Visual Acuity with a Decimal Acuity Chart.” J. Fr. Ophtalmol. 26 (6): 586-90.

[27] Pelli, D. G., and Bex, P. 2013. "Measuring Contrast Sensitivity." Vision Res. 90: 10-4. 


\section{Clinical Characterization and Frequency of Observation of Hereditary Retinal Diseases: Multicentric Study in Panama in 2012-2013}

[28] Pinckers, A., Van Aarem, A., and Keunen, J. E. E. 1993. "Colour Vision in Retinitis Pigmentosa." Int. Ophthalmol. 17 (3): 143-6.

[29] Birch, J. 2008. "Pass Rates for the Farnsworth D15 Colour Vision Test." Ophthalmic Physiol. Opt. 28 (3): 259-64.

[30] Inci Dersu, M. N. W. 2006. "Understanding Visual Fields, Part I: Goldmann Perimetry." Journal of Ophthalmic Medical Technology 2 (2): 1-10. Accessed July 5, 2016. http://www.readbag.com/jomtonline-jomt-articles-volume s-2-2-visualfields.

[31] Bertelsen, M., Jensen, H., Larsen, M., Lorenz, B., Preising, M. N., and Rosenberg, T. 2013. "Prevalence and Diagnostic Spectrum of Generalized Retinal Dystrophy in Danish Children." Ophthalmic Epidemiol. 20 (3): 164-9.

[32] Tous, H. M., and Izquierdo, N. J. 2006. "Retinitis Pigmentosa in Puerto Rico.” P. R. Health Sci. J. 25 (4): 315-8.

[33] Bocquet, B., Lacroux, A., Surget, M. O., Baudoin, C., Marquette, V., Manes, G., et al. 2013. "Relative Frequencies of Inherited Retinal Dystrophies and Optic Neuropathies in Southern France: Assessment of 21-Year Data Management." Ophthalmic Epidemiol. 20 (1): 13-25.

[34] Bertelsen, M., Jensen, H., Bregnhøj, J. F., and Rosenberg, T. 2014. "Prevalence of Generalized Retinal Dystrophy in Denmark." Ophthalmic Epidemiol. 21 (4): 217-23.

[35] Google Search. 2014. "Guidlines of the Spanish Society of Retina and Vitreous for Retinal Dystrophies." Accessed March 3, 2014. https://www.google.com/ search?q=guia + SERV + distrofias + retinianas $\&$ ie $=$ utf- $8 \& o$ $\mathrm{e}=\mathrm{utf}-8 \& \mathrm{aq}=\mathrm{t} \& \mathrm{rls}=$ org.mozilla:es-ES:official\&client=fire fox-a\&channel $=$ sb.

[36] Hamel, C. 2006. "Retinitis Pigmentosa." Orphanet. J. Rare Dis. 1 (1): 40.

[37] Rong, W., Sheng, X., and Liu, Y. 2012. “Analysis of Clinical Phenotype and Mode of Inheritance in Retinitis Pigmentosa Patients with Consanguineous Marriage." Zhonghua Yan Ke Za Zhi Chin J. Ophthalmol. 48 (10): 893-7.

[38] AAPOS (American Association for Pediatric Ophthalmology and Strabismus). 2014. "Strabismus." Accessed January 26, 2015. http://www.aapos.org/terms/conditions/100.

[39] EyeWiki. 2014. "Exotropia." Accessed May 25, 2014. http://eyewiki.aao.org/Exotropia.

[40] Park, H., Tan, C. C., Faulkner, A., Jabbar, S. B., Schmid G., Abey, J., et al. 2013. "Retinal Degeneration Increases Susceptibility to Myopia in Mice." Mol. Vis. 19: 2068-79.

[41] Alexander, K. R., Fishman, G. A., and Derlacki, D. J. 1996. "Intraocular Light Scatter in Patients with Retinitis
Pigmentosa." Vision Res. 36 (22): 3703-9.

[42] Hajali, M., and Fishman, G. A. 2009. "The Prevalence of Cystoid Macular Oedema on Optical Coherence Tomography in Retinitis Pigmentosa Patients without Cystic Changes on Fundus Examination.” Eye Lond. Engl. 23 (4): 915-9.

[43] Natarajan, S. 2011. "Retinitis Pigmentosa: A Brief Overview.” Indian J. Ophthalmol. 59 (5): 343-6.

[44] Elliott, D. B., Sanderson, K., and Conkey, A. 1990. "The Reliability of the Pelli-Robson Contrast Sensitivity Chart." Ophthalmic Physiol. Opt. J. Br. Coll. Ophthalmic Opt. Optom. 10 (1): 21-4.

[45] Mäntyjärvi, M., and Laitinen, T. 2001. "Normal Values for the Pelli-Robson Contrast Sensitivity Test." $J$. Cataract Refract. Surg. 27 (2): 261-6.

[46] Cremers, F. P., Van De Pol, D. J., Van Driel, M., Den Hollander, A. I., Van Haren, F. J., Knoers, N. V., et al. 1998. "Autosomal Recessive Retinitis Pigmentosa and Cone-Rod Dystrophy Caused by Splice Site Mutations in the Stargardt's Disease Gene ABCR." Hum. Mol. Genet. 7 (3): 355-62.

[47] EyeWiki. 2014. "Stargardt Disease/Fundus Flavimaculatus." Accessed October 23, 2014. http://eyewiki.aao.org/Stargardt_disease/Fundus_flavima culatus.

[48] Scanelli, G., Dattola, L., and Padovani, F. 1996. "Increased Risk of Autoimmune Hypothyroidism in Patients Affected by Retinitis Pigmentosa." J. Endocrinol. Invest. 19 (3): 170-4.

[49] Hamel, C. P. 2007. "Cone Rod Dystrophies." Orphanet J. Rare Dis. 2 (1): 7.

[50] Vasireddy, V., Wong, P., and Ayyagaria, R. 2010. "Genetics and Molecular Pathology of Stargardt-Like Macular Degeneration.” Prog. Retin. Eye Res. 29 (3): 191-207.

[51] NEI (National Eye Institute). "Cataracts, Statistics and Data." Accessed November 6, 2014. http://www.nei.nih.gov/eyedata/cataract.

[52] Kim, W. S., Weickert, C. S., and Garner, B. 2008. "Role of ATP-Binding Cassette Transporters in Brain Lipid Transport and Neurological Disease." J. Neurochem. 104 (5): 1145-66.

[53] OMIM (Online Mendelian Inheritance in Man). 1986. "Stargardt Disease 1; STGD1." Accessed November 2, 2014. http://www.omim.org/entry/248200.

[54] Chouchene, I., Largueche, L., Ouechtati, F., Derouiche, K., Turki, A., Abdelhak, S., et al. 2013. "Clinical Characterization of the Stargardt Disease and Molecular Exploration of the c.2041C $>$ T Mutation (ABCA4 Gene) in Tunisian Patients." Ann. Biol. Clin. (Paris) 71 (6): 645-51.

[55] Mäntyjärvi, M., and Tuppurainen, K. 1992. "Stargardt's 
Disease." Doc. Ophthalmol. 79 (1): 79-89.

[56] Doka, D. S., Fishman, G. A., and Anderson, R. J. 1982. "Refractive Errors in Patients with Fundus Flavimaculatus.” Br. J. Ophthalmol. 66 (4): 227-9.

[57] Maia-Lopes, S., Silva, E. D., Silva, M. F., Reis, A., Faria, P., and Castelo-Branco, M. 2008. "Evidence of Widespread Retinal Dysfunction in Patients with Stargardt Disease and Morphologically Unaffected Carrier Relatives." Invest. Ophthalmol. Vis. Sci. 49 (3): 1191-9.

[58] Lachapelle, P., Little, J. M., and Roy, M. S. 1989. "The Electroretinogram in Stargardt's Disease and Fundus Flavimaculatus." Doc. Ophthalmol. Adv. Ophthalmol. 73 (4): 395-404.

[59] OMIM (Online Mendelian Inheritance in Man). 1994. "Stargardt Disease 3; STGD3." Accessed November 5, 2014. http://omim.org/entry/600110.

[60] Mäntyjärvi, M., and Tuppurainen, K. 1992. "Color Vision in Stargardt's Disease.” Int. Ophthalmol. 16 (6): 423-8.

[61] Griesinger, I. B., Sieving, P. A., and Ayyagari, R. 2000. "Autosomal Dominant Macular Atrophy at 6q14 Excludes CORD7 and MCDR1/PBCRA Loci." Invest. Ophthalmol. Vis. Sci. 41 (1): 248-55.

[62] Grover, S., Alexander, K. R., Fishman, G. A., and Ryan, J. 2002. "Comparison of Intraocular Light Scatter in Carriers of Choroideremia and X-Linked Retinitis Pigmentosa." Ophthalmology 109 (1): 159-63.

[63] Noble, K. G. 1977. "Central Areolar Choroidal Dystrophy.” Am. J. Ophthalmol. 84 (3): 310-8.

[64] Wells, J., Wroblewski, J., Keen, J., Inglehearn, C., Jubb, C., Eckstein, A., et al. 1993. "Mutations in the Human Retinal Degeneration Slow (RDS) Gene Can Cause Either Retinitis Pigmentosa or Macular Dystrophy." Nat. Genet. 3 (3): 213-8.

[65] Hughes, A. E., Lotery, A. J., and Silvestri, G. 1998. "Fine Localisation of the Gene for Central Areolar Choroidal Dystrophy on Chromosome." J. Med. Genet. 35 (9): 770-2.

[66] Klevering, B. J., Van Driel, M., Van Hogerwou, A. J. M., Van De Pol, D. J. R., Deutman, A. F., Pinckers, A. J. L.
G., et al. 2002. "Central Areolar Choroidal Dystrophy Associated with Dominantly Inherited Drusen." $\mathrm{Br} . \mathrm{J}$. Ophthalmol. 86 (1): 91-6.

[67] Ouechtati, F., Belhadj Tahar, O., Mhenni, A., Chakroun, S., Chouchene, I., Oueslati, S., et al. 2009. "Central Areolar Choroidal Dystrophy Associated with Inherited Drusen in a Multigeneration Tunisian Family: Exclusion of the PRPH2 Gene and the 17p13 Locus." J. Hum. Genet. 54 (10): 589-94.

[68] Wright, K. W., and Spiegel, P. H. 2003. Pediatric Ophthalmology and Strabismus. New York: Springer.

[69] Bijveld, M. M. C., Van Genderen, M. M., Hoeben, F. P., Katzin, A. A., Van Nispen, R. M. A., Riemslag, F. C. C., et al. 2013. "Assessment of Night Vision Problems in Patients with Congenital Stationary Night Blindness." PloS One 8 (5): e62927.

[70] Bijveld, M. M. C., Florijn, R. J., Bergen, A. A. B., Van Den Born, L. I., Kamermans, M., Prick, L., et al. 2013. "Genotype and Phenotype of 101 Dutch Patients with Congenital Stationary Night Blindness." Ophthalmology 120 (10): 2072-81.

[71] Li, H., Liu, L., Xu, H., Xu, F., Jiang, R., and Sui, R. 2012. "Clinical Features of Congenital Stationary Night Blindness." Zhonghua Yi Xue Za Zhi 92 (39): 2756-9.

[72] OMIM (Online Mendelian Inheritance in Man). 1986. "Fundus Albipunctatus." Accessed February 1, 2015. http://omim.org/entry/136880.

[73] Niwa, Y., Kondo, M., Ueno, S., Nakamura, M., Terasaki, H., and Miyake, Y. 2005. "Cone and Rod Dysfunction in Fundus Albipunctatus with RDH5 Mutation: An Electrophysiological Study." Invest. Ophthalmol. Vis. Sci. 46 (4): 1480-5.

[74] Boycott, K. M., Sauvé, Y., and MacDonald, I. M. 1993. "X-Linked Congenital Stationary Night Blindness." In: GeneReviews $^{\circledR}$, edited by Pagon, R. A., Adam, M. P., Ardinger, H. H., Bird, T. D., Dolan, C. R., Fong, C. T., et al. Seattle (WA): University of Washington, Seattle; Accessed September $\quad 7,2014$. http://www.ncbi.nlm.nih.gov/books/NBK1245/. 